\title{
A simple, valid, numerical score for grading chest $x$-ray severity in adult smear-positive pulmonary tuberculosis
}

\author{
Anna P Ralph, ${ }^{1,2}$ Muhamed Ardian, ${ }^{3,4,5}$ Andri Wiguna, ${ }^{4,5}$ Graeme P Maguire, ${ }^{6}$ \\ Niels G Becker, ${ }^{2}$ Glen Drogumuller, ${ }^{7}$ Michael J Wilks, ${ }^{8}$ Govert Waramori, ${ }^{4}$ \\ Emiliana Tjitra, ${ }^{9}$ Sandjaja, ${ }^{9}$ Enny Kenagalem, ${ }^{3,10}$ Gysje J Pontororing, ${ }^{10}$ \\ Nicholas M Anstey, ${ }^{1,11}$ Paul M Kelly ${ }^{1,2}$
}

${ }^{1}$ Global Health Division, Menzies School of Health Research and Charles Darwin University, Australia

${ }^{2}$ National Centre for Epidemiology and Population Health Research, College of Medicine, Biology and Environment, Australian National University, Australia ${ }^{3}$ District Health Authority, Timika, Papua Province, Indonesia

${ }^{4}$ International SOS, Timika Papua Province, Indonesia ${ }^{5}$ Public Health \& Malaria Control Department, PT Freeport Indonesia, Papua Province, Indonesia

${ }^{6}$ School of Medicine and Dentistry, James Cook University, Queensland, Australia

${ }^{7}$ Department of Radiology, Royal Darwin Hospital, Northern

Territory, Australia

${ }^{8}$ Radiology Department, Royal

Adelaide Hospital, North

Terrace, Adelaide, South

Australia

${ }^{9}$ National Institute of Health Research and Development, Jakarta, Indonesia

${ }^{10}$ Menzies School of Health Research-National Institute of Health Research and

Development Research Program, and District Ministry of Health, Timika, Papua Province, Indonesia

${ }^{11}$ Division of Medicine, Royal Darwin Hospital, Darwin, Australia

\section{Correspondence to}

Dr Anna Ralph, International Health Division, Menzies School of Health Research, PO Box 41096, Casuarina, Northern Territory, 0811, Australia; anna.ralph@menzies.edu.au

Received 8 February 2010 Accepted 30 June 2010

\section{ABSTRACT}

Background The grading of radiological severity in clinical trials in tuberculosis (TB) remains unstandardised. The aim of this study was to generate and validate a numerical score for grading chest $x$-ray (CXR) severity and predicting response to treatment in adults with smear-positive pulmonary TB.

Methods At a TB clinic in Papua, Indonesia, serial CXRs were performed at diagnosis, 2 and 6 months in 115 adults with smear-positive pulmonary TB. Radiographic findings predictive of 2-month sputum microscopy status were used to generate a score. The validity of the score was then assessed in a second data set of 139 comparable adults with TB, recruited 4 years later at the same site. Relationships between the CXR score and other measures of TB severity were examined.

Results The estimated proportion of lung affected and presence of cavitation, but not cavity size or other radiological findings, significantly predicted outcome and were combined to derive a score given by percentage of lung affected plus 40 if cavitation was present. As well as predicting 2-month outcome, scores were significantly associated with sputum smear grade at diagnosis $(\mathrm{p}<0.001)$, body mass index, lung function, haemoglobin, exercise tolerance and quality of life $(p<0.02$ for each). In the validation data set, baseline CXR score predicted 2-month smear status significantly more accurately than did the proportion of lung affected alone. In both data sets, CXR scores decreased over time $(p<0.001)$.

Conclusion This simple, validated method for grading CXR severity in adults with smear-positive pulmonary TB correlates with baseline clinical and microbiological severity and response to treatment, and is suitable for use in clinical trials.

\section{INTRODUCTION}

Sputum smear microscopy, and culture where available, are standardised modalities for diagnosing and monitoring treatment response in pulmonary tuberculosis (TB). Chest radiography (CXR) provides useful information regarding disease extent and progress, but there is no agreed-upon, validated system for grading the severity of CXR abnormalities in bacteriologically proven pulmonary TB. Several methods were devised for this purpose at the time of early TB treatment trials, such as those described by the Madras TB Chemotherapy Centre in $1960,{ }^{1}$ Simon in $1966^{2}$ and the National TB and Respiratory Disease Association of the USA in 1969. ${ }^{3}$ Despite this, no system has been validated in predicting outcome in more than one patient population. Recent randomised controlled trials $(\mathrm{RCTs})^{4-7}$ and observational studies in adults with $\mathrm{TB}^{8-13}$ illustrate this lack of standardisation in the grading of radiological severity, with each of these studies utilising different non-validated investigatorgenerated systems to grade CXR severity.

The same problem of non-standardised radiological reporting has been recently articulated by Dawson et al relating to TB screening, who evaluated and recommended the Chest Radiographic Reading and Reporting System ${ }^{14}$ for TB screening in HIV-positive people. ${ }^{15}$ However, this and other screening tools ${ }^{16-18}$ seek to identify the presence of latent $\mathrm{TB}$ infection or active disease, and are not useful for researchers wishing to accurately document severity or response to treatment in active TB.

Problems in CXR reporting arise from the heterogeneous CXR manifestations of pulmonary TB (eg, in primary vs post primary disease, adults vs children, immunocompetent vs immunocompromised $)^{19-21}$ and to inaccuracies inherent in CXR performance and interpretation, ${ }^{2}$ including limited interobserver agreement on CXR findings. ${ }^{22}{ }^{23}$ Despite these shortcomings, the utility of CXR is well established in TB diagnosis and clinical monitoring.

Associations between radiological extent and other measures such as forced expiratory volume in $1 \mathrm{~s}\left(\mathrm{FEV}_{1}\right)$, age or multidrug-resistant (MDR)-TB have previously been identified, ${ }^{82}$ but a standard, simple, numerical score, validated against $\mathrm{TB}$ outcome, in repeated data sets, is lacking. We therefore aimed to devise a simple CXR score for use in adults with smear-positive pulmonary $\mathrm{TB}$, which predicts outcome and correlates with bacteriological and clinical severity markers, for the purpose of grading severity and monitoring treatment response in the context of TB clinical trials. We then determined the utility of the score in a separate, comparable patient population.

\section{METHODS}

\section{Study setting}

The study was conducted at a community-based TB clinic in Timika, Papua Province, Indonesia. Timika has population of $\sim 200000$ and an estimated TB incidence of 311/100 $000 .{ }^{25}$ 


\section{Participants}

Adults ( $>15$ years) diagnosed with sputum smear-positive pulmonary $\mathrm{TB}$ who gave written informed consent were eligible for enrolment in the study. Study participants were recruited during two time periods: 2003-2004 (the derivation data set) and 2008-2009 (the validation data set). The demographic, clinical and microbiological findings and outcomes in the first data set have been reported previously. ${ }^{26} 27$

\section{Chest radiography}

Standard full-size posteroanterior CXR were performed at the time of $\mathrm{TB}$ diagnosis and 2 and 6 months thereafter, with reports provided by a clinician at the field site (first data set, PMK; second data set, APR) and, additionally for the first data set, by one of two radiologists (MJW or GD). During the first data collection period, the presence of small $(1-2 \mathrm{~mm})$ or large (>2 mm) nodules, patchy or confluent consolidation, cavitation, bronchial lesions or fibrosis was reported for each of three zones (upper, mid or lower zones) in each lung. The presence of effusion or lymphadenopathy was reported, the total percentage of each lung affected by any pathology was estimated, total cavity size in millimetres was recorded and the effusion volume (percentage of lung field) was estimated. To grade the percentage of affected lung, visual estimation of the extent of opacification, cavitation or other pathology as a percentage of visible lung was made; dense opacification of a zone was graded as $100 \%$ of that zone, while patchy opacification within a zone attracted scores $<100 \%$ depending on the extent of opacification. Other remarks including presence of miliary disease were recorded. During the second data collection period, a simplified CXR report method was used (percentage lung affected, cavitation $(0,<4 \mathrm{~cm}$, $\geq 4 \mathrm{~cm}$ ), effusion $(0,<25 \%, \geq 25 \%$ of hemithorax), presence of consolidation, fibrosis, nodules, miliary disease). Reporters were blinded to HIV status, bacteriological and clinical parameters and treatment outcome.

\section{Sputum microscopy and clinical evaluations}

Baseline sputum microscopy was performed at the onsite laboratory and repeated at the reference laboratory on samples collected at 0, 2 and 6 months, and the density of acid-fast bacilli (AFB) was graded as 1,2 or $3+$ according to standard protocols. $^{26} 27$ Baseline and follow-up evaluations included: body mass index (BMI), $\mathrm{FEV}_{1}$ (spirometry performed using ML3535C, MicroLoop, MicroMedical, Chatham, UK), haemoglobin ( $\mathrm{Hb})$, measured using point-of-care HemoCue (Ängelholm, Sweden) or iSTAT (Abbott Park, Illinois, USA) tests, 6 min walk test (distance walked in 6 min on a straight walking track), measured according to American Thoracic Society guidelines, and St George's Respiratory Questionnaire (SGRQ) modified to reflect local conditions and translated into Indonesian. ${ }^{27} 28$ Standard definitions were used for nutritional category (normal, mild malnutrition, moderate malnutrition or severe malnutrition) according to $\mathrm{BMI},{ }^{29}$ and for $\mathrm{TB}$ treatment outcome at 6 months (cured, completed, transferred, defaulted, failed or died). ${ }^{30}$ Impairment in $\mathrm{FEV}_{1}$ as a percentage of predicted values was calculated using previously established local reference ranges. ${ }^{31}$

\section{Outcome measure}

The outcome measure used in this study is 2-month sputum AFB microscopy status. Two-month smear positivity has been previously shown to predict unfavourable outcomes including treatment failure and death, ${ }^{32-34}$ and determines the need for continued intensive-phase treatment versus switching to continuation-phase therapy. ${ }^{30}$ Although an imperfect predictor of outcome, ${ }^{35}$ in the absence of suitable alternatives, it remains a commonly used surrogate end point.

\section{Data analysis}

Statistical calculations were performed using Intercooled Stata 10.1 (StataCorp, College Station, Texas, USA); graphs were created in GraphPad Prism 5 (GraphPad, La Jolla, California, USA). Statistical tests were two sided, with a $p$ value of $<0.05$ indicating statistical significance. Intergroup differences in means or medians were compared using two-sample t tests, Wilcoxon rank sum tests, analysis of variance or Kruskal-Wallis tests as appropriate.

Agreement between reporters in the derivation data set was tested using the concordance coefficients, $\rho_{c}$ for continuous variables or the kappa statistic for categorical variables. Prevalence-adjusted, bias-adjusted kappa values were calculated according to the method described by Byrt et al. ${ }^{36}$ Kappa values were interpreted according to guidelines given by Landis and $\mathrm{Koch}^{37}$ (kappa $\leq 0.00$, poor; 0.00-0.20, slight; 0.21-0.40, fair; 0.41-0.60, moderate; $0.61-0.80$, substantial; $0.81-1.00$, almost perfect).

The relationships between radiographic findings and clinical outcome were examined by multivariable regression analysis, using a forward stepwise approach in which any radiological variable found to be significant $(p<0.05)$ in univariate analysis was included in the initial model. Goodness of fit of final models was assessed using the Hosmer-Lemeshow test and compared using the likelihood ratio test. The weighting for a numerical radiological score was derived from the regression coefficients. Its ability to predict outcome in the validation data set was determined using receiver operator characteristics (ROC; area under the curve (AUC)). The relationships between this score and demographic, biological and clinical variables were determined in data sets 1 and 2 using regression models using the same principles.

\section{Ethics}

Approval was granted by the ethics committees of the National Institute of Health Research and Development (Jakarta, Indonesia), Menzies School of Health Research (Darwin, Australia) and the Australian National University (Canberra, Australia). Written informed consent was obtained from participants in Indonesian or an appropriate Papuan language.

\section{RESULTS}

Characteristics of study participants in the two data collection phases are shown in table 1 . All participants had smear-positive pulmonary $\mathrm{TB}$ ( $\geq 2$ AFB smear-positive sputum samples); the result of an additional sample provided for microscopy and culture on the day of treatment commencement is reported here. This was negative in $5.7 \%$ and $7.2 \%$ of participants in the two data sets, respectively despite their previous samples being positive. Initial smear grade predicted the likelihood of smear conversion by 2 months. In the derivation data set, failure to convert to smear negative by 2 months was observed in $60.9 \%$ of patients with a baseline smear grade of 3 and in $38.7 \%$ of patients with a baseline smear grade of $<3+(p=0.051)$. In the validation data set, failure to convert to smear negative by 2 months was observed in $48.4 \%$ of patients with a baseline smear grade of 3 and in $11.8 \%$ of patients with a baseline smear grade of $<3+(p<0.001)$.

CXR reports were available at baseline, 2 and 6 months for 112, 76 and 76 study participants in the first data set, and 136, 93 and 76 study participants in the second data set (incomplete in the second data set as 30 of 139 had not yet completed 
Table 1 Study participant characteristics

\begin{tabular}{|c|c|c|}
\hline & Derivation data set & Validation data set \\
\hline \multicolumn{3}{|l|}{ Demographic details } \\
\hline Number & 115 & 139 \\
\hline Age in years: median (range) & $30(17-69)$ & $27(15-65)$ \\
\hline Female gender, $\mathrm{n}(\%)$ & $33(28.7)$ & $48(34.5)$ \\
\hline Papuan ethnicity, n (\%) & $57(49.6)$ & $66(47.5)$ \\
\hline Smokers, n (\%) & $38(33.0)$ & $41(29.5)$ \\
\hline HIV positive: no./no. tested (\%) & $5 / 112(4.5)$ & $16 / 121(13.2)$ \\
\hline MDR-TB, n (\%) & $2(1.7)$ & $2(1.4)$ \\
\hline \multicolumn{3}{|l|}{ Baseline clinical findings } \\
\hline BMI, median (range), $\mathrm{kg} / \mathrm{m}^{2}$ & $18.6(14.2-25.2)$ & $19.0(12.9-32.5)$ \\
\hline Haemoglobin, median (range), g/dl & $11.2(6.8-18.0)$ & $12.2(7.1-16.0)$ \\
\hline $\mathrm{FEV}_{1}:$ median (range), litres & $1.76(0.49-4.12)$ & $1.70(0.59-3.56)$ \\
\hline SGRO total score, median (range) & $45.3(2.5-83.5)$ & $37.8(5.2-91.9)$ \\
\hline 6 min walk distance, median (range) $\mathrm{m}$ & $405(185-625)$ & $410(20-612)$ \\
\hline \multicolumn{3}{|c|}{ Sputum AFB smear grade at diagnosis $\mathrm{n}(\%)$} \\
\hline $0^{*}$ & $6(5.7)$ & $10(7.2)$ \\
\hline Scanty or $1+$ & $25(23.6)$ & $65(45.7)$ \\
\hline $2+$ & $28(26.4)$ & $35(25.2)$ \\
\hline $3+$ & $47(44.3)$ & $29(20.9)$ \\
\hline \multicolumn{3}{|l|}{ 2-month smear status $\mathrm{n}(\%)$} \\
\hline Positive & $25(21.8)$ & $31(22.3)$ \\
\hline Negative & $81(70.4)$ & $95(68.4)$ \\
\hline No result available & $9(7.8)$ & $13(9.3)$ \\
\hline \multicolumn{3}{|l|}{ 6-month outcome n (\%) } \\
\hline Cured/completed & $88(76.5)$ & $92(66.2)$ \\
\hline Died & $3(2.6)$ & $2(1.4)$ \\
\hline Failed & $2(1.7)$ & $1(0.7)$ \\
\hline Default & $13(11.3)$ & $8(5.8)$ \\
\hline Transferred & $9(7.8)$ & $6(4.3)$ \\
\hline 6 months not yet completed & 0 & $30(21.6)$ \\
\hline
\end{tabular}

*All study participants had at least two prior smear-positive sputum samples; some are reported as negative since this result pertains to the additional spot specimen provided at enrolment into the study (see the methods section).

$A F B$, acid-fast bacilli; BMI, body mass index; $F_{1}$, forced expiratory volume in $1 \mathrm{~s}$; MDR-TB, multidrug-resistant tuberculosis; SGRQ, St George's Respiratory Questionnaire.

6 months) (table 2). Reasons for missing CXR included patient failure to attend (died, defaulted or transferred prior to appointment), inability to obtain CXR (eg, electricity failure), CXR date $>3$ weeks before or after the due follow-up date, or CXR unavailable for reporting.

\section{Agreement on radiological abnormalities}

Agreement between reporters on radiological abnormalities in the derivation data set is shown in table 3. Agreement was relatively low overall. More substantial agreement was achieved for some variables after adjusting kappa values for variable prevalence and reporter bias.

\section{Development of score using the training data set}

Two-month sputum smear status in the initial data set $(n=115)$ was significantly predicted in univariate logistic regression models by the presence of baseline cavitation (OR 3.26, 95\% CI 1.11 to 9.56 ) and the total percentage of lung affected (OR 1.9, $95 \%$ CI 1.3 to 2.7 , calculated per each $20 \%$ increment of affected lung), but not by cavitation size, presence or number of nodules, fibrosis, effusion or lymph nodes (table 4).

The relationships between those radiological findings which were independently predictive of 2-month outcome (cavitation and percentage of lung affected) and baseline clinical and bacteriological measures were then examined. Cavitary disease on CXR at TB diagnosis was significantly associated with higher baseline AFB density in sputum (ie, smear microscopy grade) $\left(p=0.007, \chi^{2}\right.$ test for trend), and people with cavitary disease
Table 2 Chest radiograph (CXR) results

\begin{tabular}{|c|c|c|}
\hline & $\begin{array}{l}\text { Derivation } \\
\text { data set }\end{array}$ & $\begin{array}{l}\text { Validation } \\
\text { data set }\end{array}$ \\
\hline \multicolumn{3}{|l|}{ Number of CXR available for report } \\
\hline Baseline & 112 & 136 \\
\hline 2 months & 76 & 93 \\
\hline 6 months & 76 & $76^{*}$ \\
\hline \multicolumn{3}{|l|}{ Baseline radiological findings } \\
\hline Consolidation, n (\%) & $106(94.6)$ & $104(76.5)$ \\
\hline Cavitation, n (\%) & $71(63.4)$ & $77(56.6)$ \\
\hline Effusion, n (\%) & $37(33.0)$ & $24(17.3)$ \\
\hline Miliary, n (\%) & $2(1.7)$ & $5(3.7)$ \\
\hline Nodules, n (\%) & $83(72.2)$ & $29(21.3)$ \\
\hline Fibrosis, n (\%) & $62(55.3)$ & $10(7.4)$ \\
\hline Proportion of lung affected, median \% (range) & $35(0-100)$ & $41.5(0-100)$ \\
\hline \multicolumn{3}{|l|}{ CXR weighted score characteristics } \\
\hline Baseline score, median (range) & $65(5-130)$ & $69(0-140)$ \\
\hline 2 month score, median (range) & $35(0-130)$ & $29(0-140)$ \\
\hline 6 month score, median (range) & $10(0-105)$ & $10(0-115)$ \\
\hline Median score decrease in 2 months, \% & 27.3 & 38.8 \\
\hline Median score decrease in 6 months, $\%$ & 73.1 & 80.2 \\
\hline \multicolumn{3}{|c|}{ Baseline score by smear grade at diagnosis median (range) } \\
\hline 0 & $15(0.5-70)$ & $10(0-133)$ \\
\hline Scanty or $1+$ & $50(0-110)$ & $66(0-140)$ \\
\hline $2+$ & $70(6.5-135)$ & $76(11-140)$ \\
\hline $3+$ & $80(10-140)$ & $85(4-140)$ \\
\hline
\end{tabular}

had worse lung function, with a mean percentage predicted $\mathrm{FEV}_{1}$ of 59.0 (95\% CI 54.4 to 63.6) in cavitary disease versus 68.7 (95\% CI 60.6 to 76.7$)$ in non-cavitary disease $(p=0.03$, two-sample t test). People with cavitary disease had slightly lower BMI (18.5, 95\% CI 18.0 to 19.1) compared with those with non-cavitary disease $\left(19.2 \mathrm{~kg} / \mathrm{m}^{2}, 95 \%\right.$ CI 18.5 to 20.0$)$, but this difference was not statistically significantly (mean betweengroup difference $0.70 \mathrm{~kg} / \mathrm{m}^{2}, 95 \% \mathrm{CI}-0.18$ to 1.59 ). No significant associations were identified between cavitary disease and exercise tolerance ( 6 min walk distance), quality of life (SGRO total or individual domain scores) or $\mathrm{Hb}$.

The amount (\%) of lung affected significantly predicted all clinical and laboratory variables. Specifically, greater proportions of affected lung were significantly associated with decreasing BMI category ( $p=0.002$, Kruskal-Wallis test), lung function category

Table 3 Agreement on radiological findings, derivation data set

\begin{tabular}{|c|c|c|c|}
\hline $\begin{array}{l}\text { Inter-rater agreement } \\
\text { among dichotomous } \\
\text { variables }\end{array}$ & Kappa & $\begin{array}{l}\text { Prevalence- } \\
\text { adjusted, } \\
\text { bias-adjusted } \\
\text { kappa }\end{array}$ & $\begin{array}{l}\text { Interpretation } \\
\text { of prevalence- } \\
\text { adjusted, } \\
\text { bias-adjusted } \\
\text { kappa }\end{array}$ \\
\hline Presence of patchy consolidation & 0.20 & 0.70 & Substantial \\
\hline Presence of confluent consolidation & 0.33 & 0.31 & Fair \\
\hline Presence of any consolidation & 0.24 & 0.81 & Almost perfect \\
\hline Presence of small nodules & 0.12 & 0.06 & Slight \\
\hline Presence of large nodules & 0.09 & 0.09 & Slight \\
\hline Presence of any nodules & 0.19 & 0.19 & Slight \\
\hline Presence of fibrosis & 0.08 & 0.06 & Slight \\
\hline Presence of cavitation & 0.33 & 0.37 & Fair \\
\hline Presence of effusion & -0.35 & -0.61 & Poor \\
\hline Presence of lymphadenopathy & 0.01 & -0.09 & Poor \\
\hline $\begin{array}{l}\text { Concordance among } \\
\text { continuous variables }\end{array}$ & \multicolumn{3}{|c|}{$\begin{array}{l}\text { 95\% limits of agreement } \\
\text { (Bland and Altman) }\end{array}$} \\
\hline Total amount $(\%)$ of lung affected & 0.85 & \multicolumn{2}{|c|}{-28.20 to $22.46 \%$} \\
\hline Extent of cavity size (mm) & 0.69 & \multicolumn{2}{|c|}{-56.62 to $50.66 \mathrm{~mm}$} \\
\hline
\end{tabular}


Table 4 Relationship between radiological and biological parameters and 2-month sputum acid-fast bacilli density in the derivation data set, showing results of univariate logistic regression analyses*

\begin{tabular}{llll}
\hline & Odds ratio & 95\% CI & p Value \\
\hline $\begin{array}{l}\text { Radiological parameters } \\
\text { Presence of any consolidation }\end{array}$ & & & \\
$\quad$ Per 1\% increments & 1.30 & 0.14 to 12.18 & 0.001 \\
$\quad$ Per 20\% increments & 1.90 & 1.30 to 2.68 & 0.001 \\
Cavitary disease & 3.26 & 1.11 to 9.56 & 0.032 \\
Effusion & 1.50 & 0.59 to 3.82 & 0.590 \\
Nodules & 1.17 & 0.41 to 3.32 & 0.773 \\
Fibrosis & 2.23 & 0.86 to 5.79 & 0.097 \\
Lymphadenopathy & 1.43 & 0.57 to 3.56 & 0.448
\end{tabular}

*OR was unable to be calculated for the two instances of military disease (both had negative sputum at 2 months).

$(\mathrm{p}<0.001$, Kruskal-Wallis test), 6 min walk distance $(0=0.001$, linear regression) and $\mathrm{Hb}$ in males ( $\mathrm{p}=0.003$, linear regression), though not in females $(p=0.4)$. A greater proportion of lung affected on the baseline CXR was also significantly associated with SGRQ total scores $(p<0.001$, linear regression) and with sputum smear grade at diagnosis ( $<<0.001$, Kruskal-Wallis test).

To create a CXR score, cavitation and percentage of lung affected were included as independent variables in a logistic regression model for 2 -month sputum smear status. The model containing both variables was significantly better than the model containing cavitaion alone (likelihood ratio test $\mathrm{p}<0.001$ ) or proportion of lung affected alone (likelihood ratio test $p=0.016$ ) at predicting 2-month sputum smear status. Regression coefficients were 0.03167 for proportion of lung affected and 1.26151 for presence/absence of cavitation, indicating a relative weighting of 40.27 for cavitation $(1.26151 \div 0.03167)$, thereby generating an equation for the weighted score as follows:

CXR score $=$ proportion of total lung affected $(\%)$

$$
+40 \text { if cavitation present }
$$

\section{CXR score results}

CXR score characteristics are shown in table 2 and figures $1-4$. Scores did not significantly differ according to sex, ethnicity or smoking status ( $p>0.05$, two-sample $t$ tests), and were not

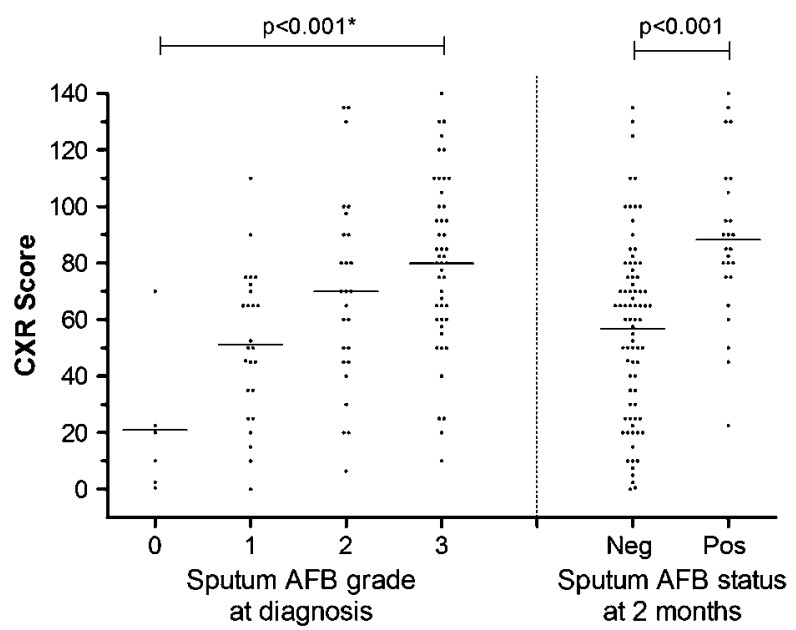

${ }^{*} p<0.001$ calculated using one-way analysis of variance for sputum grade at diagnosis; $p<0.001$ calculated using 2-sample t-test for sputum status at 2 months. Bars represent mean score.

Figure 1 Chest $x$-ray (CXR) score at enrolment according to sputum acid-fast bacilli (AFB) grade at diagnosis and 2 months (derivation data set).

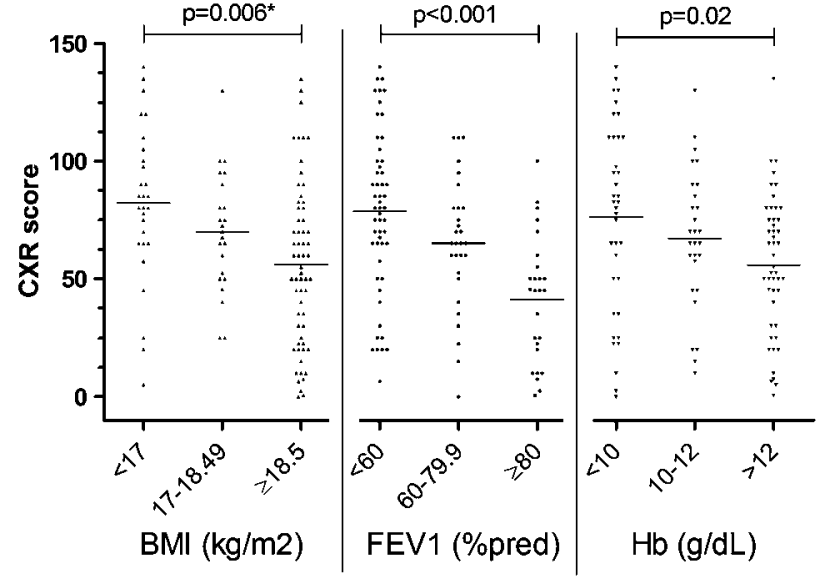

*All $p$ values calculated from one-way analyses of variance. Horizontal bars represent mean score.

Figure 2 Chest x-ray (CXR) score according to body mass index (BMI), percentage of predicted forced expiratory volume in $1 \mathrm{~s}\left(\mathrm{FEV}_{1}\right)$ and haemoglobin $(\mathrm{Hb})$ at diagnosis (derivation data set).

significantly associated with age in univariate or multivariate analyses. Mean baseline CXR score in people with unfavourable (positive) 2-month outcomes was significantly higher (88.2; 95\% CI 76.5 to 99.9) than in those with a favourable outcome (56.8; $95 \%$ CI 49.7 to 64.0 ), but the range of scores in each smear grade was wide (figure 1). Scores were also significantly associated with baseline microscopy grade (figure 1). CXR scores were inversely related to $\mathrm{BMI}, \mathrm{FEV}_{1}, \mathrm{Hb}$ and 6 min walk distance, were directly related to SGRQ total score (higher SGRQ scores indicate worse quality of life) and significantly decreased over time (figures 2-4).

\section{Performance of score using the validation data set}

The weighted score calculated for the new data set showed similar characteristics (table 2), including a median baseline score of 69, no significant relationship with demographic factors, significant positive association with baseline smear grade $(\mathrm{p}=0.009$, Kruskal-Wallis test $)$ and the same relationships as

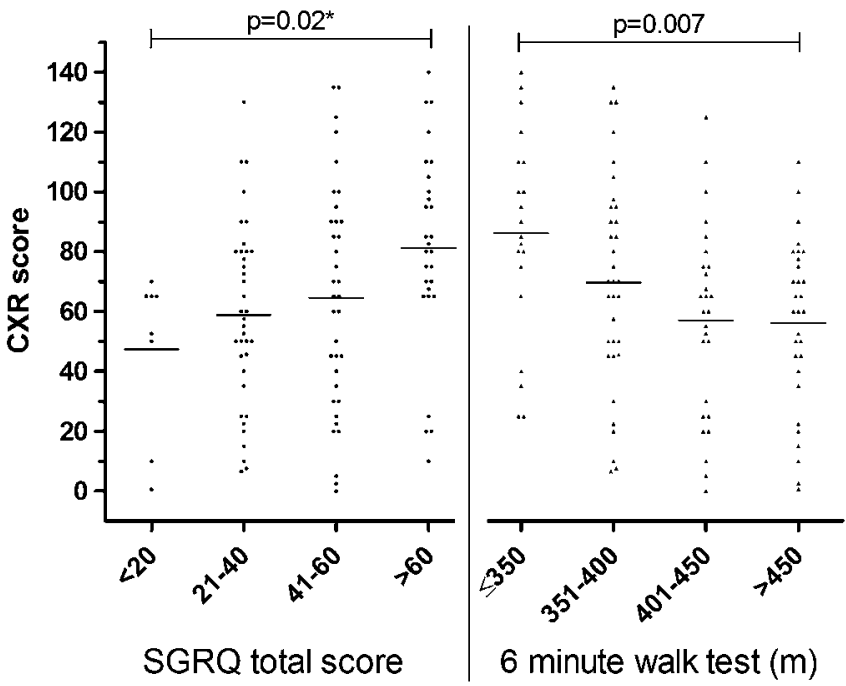

${ }^{*}$ Both $p$ values calculated using one-way analyses of variance. Horizontal bars represent mean score.

Figure 3 Chest x-ray (CXR) score according to St George's Respiratory Questionnaire (SGRQ) (total score and 6 minute walk distance at tuberculosis diagnosis (derivation data set). 
Figure 4 Chest x-ray (CXR) score at diagnosis, 8 weeks (end of intensive treatment phase) and 24 weeks (end of treatment).
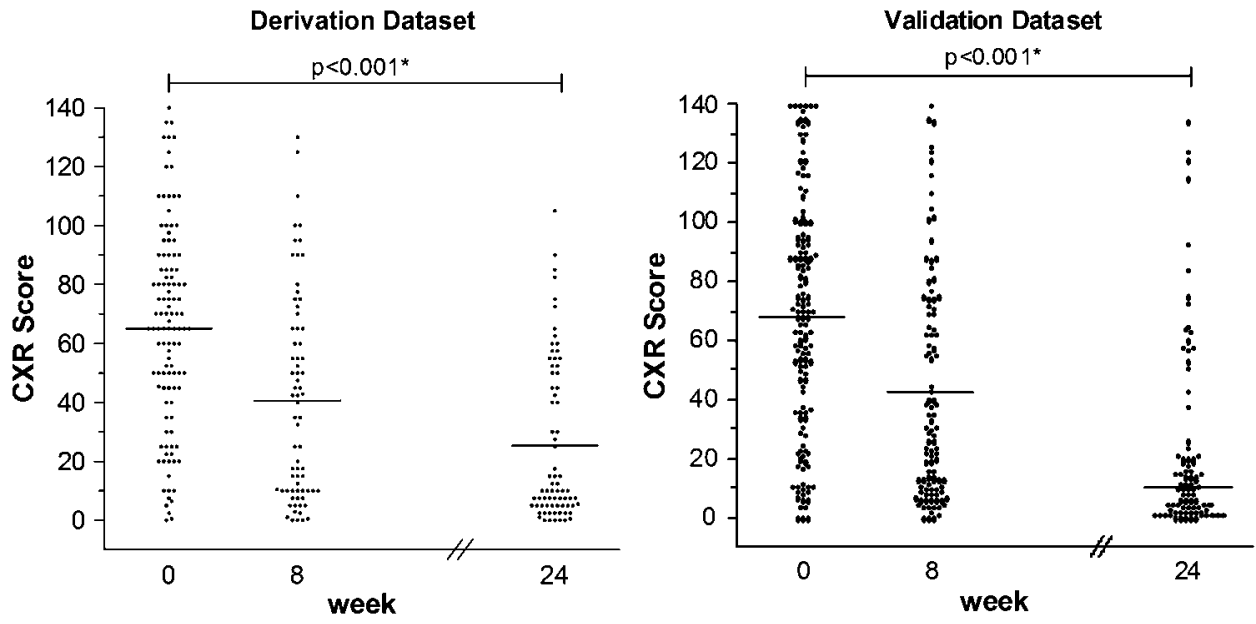

${ }^{*} p$ values calculated from Kruskal-Wallis tests. Horizontal bars represent median CXR scores. were found in the initial data set between CXR score and each of the clinical/laboratory measures (BMI, Hb, FEV ${ }_{1}, \mathrm{SGRO}$ total score and 6 min walk distance; $p<0.05$ in each case).

Comparing ROC scores to predict outcome, the weighted CXR score (AUC 0.75) was significantly better at predicting 2-month smear status than the percentage lung affected alone (ROC 0.69; $\mathrm{p}=0.013, \chi^{2}$ test; figure 5). The optimal cut-off point for weighted CXR score (value furthest from the diagonal) was 71 , at which value the sensitivity for predicting a positive sputum smear status at 2 months was $80 \%$ (95\% CI 61.4 to 92.3 ) and specificity $67.7 \%$ (95\% CI 57.3 to 77.1$)$. Comparative sensitivity and specificity values are shown in table 5 .

\section{DISCUSSION}

The current need for a universal and standard system for reporting CXR in pulmonary $\mathrm{TB}$ is acknowledged. ${ }^{38}$ In order to grade CXR severity and assess radiological treatment response, we have derived a simple equation from radiographic parameters from adults with smear-positive pulmonary $\mathrm{TB}$ that predicts smear positivity at 2 months and provides a single numerical

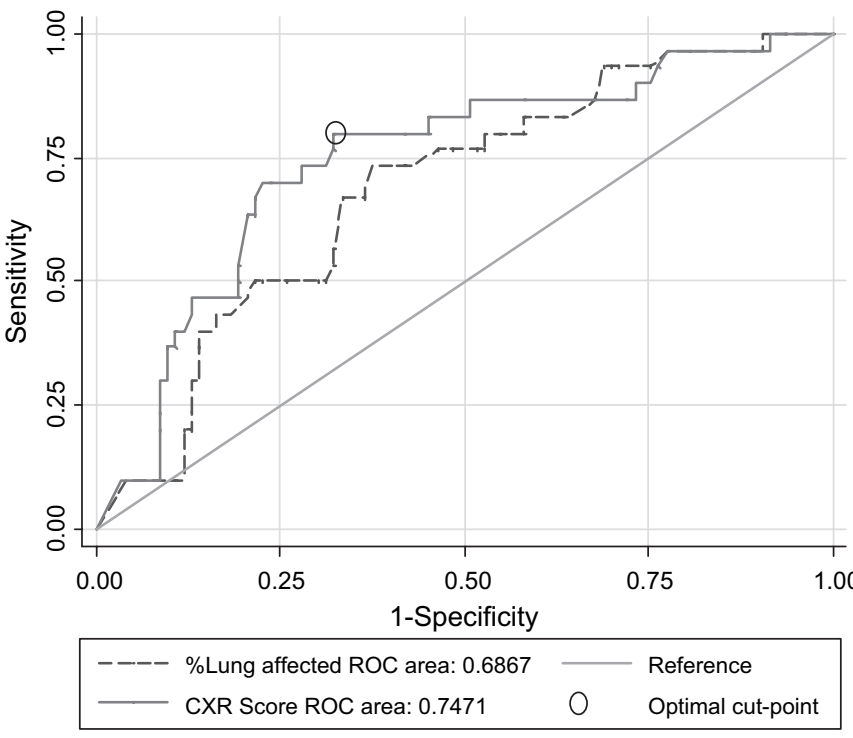

Figure 5 Receiver operator characteristics (ROC) for weighted chest $x$-ray (CXR) score (\% lung affected +40 if cavitation present) (solid line) compared with percentage of lung affected (dashed line) as predictors of 2-month smear status (positive or negative). Optimal cut-off point for score $($ circle $)=71$. score for each CXR. The score shows good correlation with baseline bacteriological and clinical severity markers, and is sensitive to changes over time. The score performs better than its individual components: it was significantly better at predicting outcome than was the percentage of lung affected alone, and was significantly associated with a broader range of baseline severity measures (BMI, $\mid \mathrm{Hb}$, exercise tolerance and quality of life) than presence of cavitation alone. Advantages of this method are that CXR assessment does not require aids, grids or rulers, and it is derived by fitting a statistical model to outcome data rather than by assigning points based on assumed relative importance of radiographic pathologies. It has been

Table 5 Sensitivity and specificity of baseline radiological and bacteriological findings in predicting outcome (2-month sputum acid-fast bacilli (AFB) status)

\begin{tabular}{|c|c|c|}
\hline & Sensitivity & Specificity \\
\hline \multicolumn{3}{|c|}{ Radiological parameters at diagnosis } \\
\hline \multicolumn{3}{|c|}{ Derivation data set } \\
\hline \multicolumn{3}{|l|}{ Total amount of lung affected } \\
\hline $20 \%$ & 96 & 35.9 \\
\hline $\begin{array}{l}37.5 \% \text { (optimal cut-off } \\
\text { point) }\end{array}$ & 84 & 68.0 \\
\hline $80 \%$ & 20 & 89.7 \\
\hline Cavitation & 80 & 44.9 \\
\hline \multicolumn{3}{|l|}{ Score } \\
\hline 20 & 100 & 18.0 \\
\hline 72.5 (optimal cut-off point) & 80 & 71.2 \\
\hline 130 & 8 & 98.7 \\
\hline \multicolumn{3}{|l|}{ Validation data set } \\
\hline \multicolumn{3}{|l|}{ Total amount of lung affected } \\
\hline $20 \%$ & 96.7 & 22.6 \\
\hline $\begin{array}{l}42.0 \% \text { (optimal cut-off } \\
\text { point) }\end{array}$ & 73.3 & 62.4 \\
\hline $80 \%$ & 30 & 87.1 \\
\hline Cavitation & 83.3 & 52.7 \\
\hline \multicolumn{3}{|l|}{ Score } \\
\hline 20 & 96.7 & 22.6 \\
\hline 71 (optimal cut-off point) & 80 & 67.7 \\
\hline 130 & 10 & 91.4 \\
\hline \multicolumn{3}{|c|}{ Bacteriological parameters: baseline smear grade } \\
\hline \multicolumn{3}{|c|}{ Derivation data set } \\
\hline AFB density* & 60.9 & 61.3 \\
\hline \multicolumn{3}{|l|}{ Validation data set } \\
\hline AFB density * & 48.4 & 88.2 \\
\hline
\end{tabular}

${ }^{*}$ Baseline AFB density (smear grade) dichotomised as $\leq 2$ or 3 . 
validated in an independent data set, and offers a single, standardised solution where there are currently multiple unvalidated methods in use.

The proportion of lung affected and/or cavitation feature as the most important measures in many TB CXR grading methods. ${ }^{1-57}$ Cavitation is well recognised to correlate with bacillary load. ${ }^{7} 39$ We confirmed the association between cavitation and bacteriological measures (baseline and 2-month sputum smear status), and additionally showed cavitary disease to be predictive of worse lung function. The proportion of lung affected was associated with both bacteriological and a range of clinical measures.

This score was derived in adult patients with TB with smearpositive pulmonary disease, in a setting with relatively low rates of HIV-TB co-infection and MDR-TB. The score requires further evaluation in populations with high HIV prevalence, in whom CXR findings characteristic of HIV-TB co-infection (subtle or absent pathology, non-cavitary disease, lower lobe infiltrates, hilar lymphadenopathy and pleural effusion) ${ }^{20} 40$ may mean that a differently weighted score is needed. Nevertheless, the score remained valid and applicable in the newer data set in which HIV-TB co-infection rates were higher (13\%); the rise in HIV prevalence may account for some of the differences observed between the two data sets. The presence of MDR-TB would not be expected to alter radiographic patterns, other than being associated potentially with higher scores and smaller incremental improvements over time.

Potential limitations of the study include the use of 2 month smear status as an outcome measure (rather than a longer term measure such as 6-month outcome or recurrence).

The absence of suitable biomarkers or other surrogate end points in TB research is readily acknowledged, and recent estimates derived from meta-analysis found a sensitivity of only $57 \%$ and specificity of $81 \%$ for 2 -month smear status in predicting treatment failure. ${ }^{35}$ Nevertheless, until more suitable measures become available, 2-month smear status remains a suitable outcome measure. ${ }^{30} 32-34$

Another limitation was the inherent problem of limited inter-rater agreement in CXR assessment. The low rates of clinician-radiologist agreement between reporters on CXR findings identified in the derivation data set are not unusual, with only fair or poor agreement between radiologists and clinicians also being reported elsewhere. ${ }^{22} 23$ This emphasises the importance of using simple rather than complex scores and ensuring individuals allocating CXR scores participate in continuing education to maximise agreement. The score derived from radiologist CXR evaluation in the first data set is simple. Moreover, it was shown to be valid in the second data set when used by an independent TB clinician, rather than a radiologist, confirming its practical utility in a clinical and trial setting. Some systematic differences in CXR results were noted between the two data sets; while this may represent systematic difference in reporting styles, the findings are in keeping with the possibility of less severe disease in the validation data set, as indicated by their lower bacillary burden (with slides read by the same senior laboratory technician during both data collection periods).

\section{CONCLUSION}

In summary, we have derived and validated a simple method for grading CXR severity in adults with smear-positive pulmonary TB that predicts baseline clinical and microbiological severity and response to treatment in two separate patient populations. Although finer discriminatory accuracy might be achieved by collecting more detailed CXR findings (such as cavity size), our data did not indicate this. This method can be used where a numerical score is required for the purpose of comparing radiographic severity between adults with smear-positive pulmonary $\mathrm{TB}$, and to monitor an individual's improvement over time, such as in clinical trials of drug efficacy in TB.

Acknowledgements We thank the following for their support and assistance: Dr M Okoseray, Pak Penias and Pak E Meokbun and the Timika District Health Authority; Dr Dina Bisara Lolong and Ibu Meryani Girsang and the National Institute of Health Research and Development, Jakarta; Dr P Penttinen, Dr M Bangs and Dr M Stone, Public Health \& Malaria Control (PHMC) and International SOS; Pak Istanto and PHMC laboratory staff; Pak J Lempoy and Timika TB clinic staff; Dr P. Sugiarto and Mimika Community Hospital (RSMM); Natalia Dwi Haryanti, Sri Hasmunik, Sri Rahayu, G Bellatrix and clinical and laboratory staff, NIHRD-MSHR Timika research programme; Mr R Lumb and Dr I Bastian at the Institute of Medical and Vetinerary Science; and Associate Professor R Price, MSHR.

Funding Australian Respiratory Council, the Royal Australasian College of Physicians (Covance award), Australian National Health and Medical Research Council.

\section{Competing interests None.}

Ethics approval This study was conducted with the approval of the Human Research Ethics Committees of the NT Department of Health \& Families and Menzies School of Health Research, Australia, the Australian National University, and the National Institute for Health Research and Development, Indonesia.

Provenance and peer review Not commissioned; externally peer reviewed.

\section{REFERENCES}

1. Tuberculosis Chemotherapy Centre Madras. A concurrent comparison of isoniazid plus PAS with three regimens of isoniazid alone in the domiciliary treatment of pulmonary tuberculosis in South India. Bull World Health Org 1960;23:535-85.

2. Simon G. Radiology in epidemiological studies and some therapeutic trials. BMJ 1966;2:491-4.

3. Falk A, O'Connor J, Pratt P. Classification of pulmonary tuberculosis. In: Falk A, O'Connor JB, Pratt PC, et al, eds. Diagnostic standards and classification of tuberculosis. Vol 12. New York, NY: National Tuberculosis and Respiratory Disease Association, 1969:68-76.

4. Diacon AH, Pym A, Grobusch M, et al. The diarylquinoline TMC207 for multidrug-resistant tuberculosis. N Engl J Med 2009;360:2397-405.

5. Burman WJ, Goldberg S, Johnson JL, et al. Moxifloxacin versus ethambutol in the first 2 months of treatment for pulmonary tuberculosis. Am J Respir Crit Care Med 2006;174:331-8

6. Katiyar SK, Bihari S, Prakash S, et al. A randomised controlled trial of high-dose isoniazid adjuvant therapy for multidrug-resistant tuberculosis. Int J Tuberc Lung Dis 2008;12:139-45

7. Benator D, Bhattacharya M, Bozeman L, et al. Rifapentine and isoniazid once a week versus rifampicin and isoniazid twice a week for treatment of drugsusceptible pulmonary tuberculosis in HIV-negative patients: a randomised clinical trial. Lancet 2002;360:528-34.

8. Heo EY, Chun EJ, Lee $\mathrm{CH}$, et al. Radiographic improvement and its predictors in patients with pulmonary tuberculosis. Int $J$ Infect Dis 2009;13:e371-6.

9. Mankatittham W, Likanonsakul S, Thawornwan U, et al. Characteristics of HIV-infected tuberculosis patients in Thailand. Southeast Asian J Trop Med Public Health 2009; 40:93-103.

10. Lawson L, Yassin MA, Thacher TD, et al. Clinical presentation of adults with pulmonary tuberculosis with and without HIV infection in Nigeria. Scand J Infect Dis 2008:40:30-5

11. Idh J, Westman A, Elias D, et al. Nitric oxide production in the exhaled air of patients with pulmonary tuberculosis in relation to HIV co-infection. BMC Infect Dis 2008:8:146.

12. Thorson A, Long NH, Larsson LO. Chest X-ray findings in relation to gender and symptoms: a study of patients with smear positive tuberculosis in Vietnam. Scand J Infect Dis 2007:39:33-7.

13. Liam CK, Lim KH, Srinivas $P$, et al. Hypercalcaemia in patients with newly diagnosed tuberculosis in Malaysia. Int J Tuberc Lung Dis 1998;2:818-23.

14. Den Boon S, Bateman ED, Enarson DA, et al. Development and evaluation of a new chest radiograph reading and recording system for epidemiological surveys of tuberculosis and lung disease. Int J Tuberc Lung Dis 2005;9:1088-96.

15. Dawson R, Masuka P, Edwards DJ, et al. Chest radiograph reading and recording system: evaluation for tuberculosis screening in patients with advanced HIV Int J Tuberc Lung Dis 2010;14:52-8.

16. Gatner EM, Burkhardt KR. Correlation of the results of X-ray and sputum culture in tuberculosis prevalence surveys. Tubercle. 1980;61:27-31.

17. Linh NN, Marks GB, Crawford AB. Radiographic predictors of subsequent reactivation of tuberculosis. Int J Tuberc Lung Dis 2007:11:1136-42.

18. World Health Organization. Assessing tuberculosis prevalence through population-based surveys. WHO, Western Pacific Region 2007 [October 2009] http://www.wpro.who.int/publications/PUB_978+92+9061+314+5.htm. 
19. Andreu J, Caceres J, Pallisa E, et al. Radiological manifestations of pulmonary tuberculosis. Eur J Radiol 2004;51:139-49.

20. Raviglione MC, Narain JP, Kochi A. HIV-associated tuberculosis in developing countries: clinical features, diagnosis, and treatment. Bull World Health Organ 1992; 70:515-26.

21. Marais BJ, Gie RP, Schaaf HS, et al. A proposed radiological classification of childhood intra-thoracic tuberculosis. Pediatr Radiol 2004;34:886-94.

22. Balabanova $\mathbf{Y}$, Coker $\mathrm{R}$, Fedorin $\mathrm{I}$, et al. Variability in interpretation of chest radiographs among Russian clinicians and implications for screening programmes: observational study. BMJ 2005;331:379-82.

23. Zellweger $\mathbf{J P}$, Heinzer $\mathrm{R}$, Touray $\mathrm{M}$, et al. Intra-observer and overall agreement in the radiological assessment of tuberculosis. Int J Tuberc Lung Dis 2006;10:1123-6.

24. Plit ML, Anderson R, Van Rensburg CE, et al. Influence of antimicrobial chemotherapy on spirometric parameters and pro-inflammatory indices in severe pulmonary tuberculosis. Eur Respir J 1998;12:351-6.

25. Ardian M, Meokbun E, Siburian L, et al. A public-private partnership for TB control in Timika, Papua Province, Indonesia. Int J Tuberc Lung Dis 2007;11:1101-7.

26. Kelly PM, Ardian M, Waramori G, et al. A community-based TB drug susceptibility study in Mimika District, Papua Province, Indonesia. Int J Tuberc Lung Dis 2006:10:167-71.

27. Maguire GP, Anstey NM, Ardian M, et al. Pulmonary tuberculosis, impaired lung function, disability and quality of life in a high-burden setting. Int J Tuberc Lung Dis 2009:13:1500-6.

28. Jones PW, Quirk FH, Baveystock CM, et al. A self-complete measure of health status for chronic airflow limitation. The St. George's Respiratory Questionnaire. Am Rev Respir Dis 1992;145:1321-7.

29. World Health Organization. Management of severe malnutrition: a manual for physicians and other senior health workers. Geneva: 1999[December 2009]. http://whqlibdoc.who.int/hq/1999/a57361.pdf.
30. World Health Organization. Treatment of tuberculosis: guidelines for national programmes. WHO/CDS/TB/2003.313. Geneva, Switzerland: WHO, 2003.

31. Handojo T, Anstey N, Kelly P, et al. Normal spirometry, gas transfer and lung volume values in Papua, Indonesia. Southeast Asian J Trop Med Public Health 2006;37:571-7.

32. Wang JY, Lee LN, Yu CJ, et al. Factors influencing time to smear conversion in patients with smear-positive pulmonary tuberculosis. Respirology 2009;14:1012-19.

33. Rieder HL. Sputum smear conversion during directly observed treatment for tuberculosis. Tuber Lung Dis. 1996;77:124-9.

34. Ramarokoto H, Randriamiharisoa H, Rakotoarisaonina A, et al. Bacteriological follow-up of tuberculosis treatment: a comparative study of smear microscopy and culture results at the second month of treatment. Int $J$ Tuberc Lung Dis 2002;6:909-12.

35. Horne DJ, Royce SE, Gooze L, et al. Sputum monitoring during tuberculosis treatment for predicting outcome: systematic review and meta-analysis. Lancet Infect Dis 2010;10:387-94.

36. Byrt T, Bishop J, Carlin JB. Bias, prevalence and kappa. J Clin Epidemiol 1993; 46:423-9.

37. Landis JR, Koch GG. The measurement of observer agreement for categorical data. Biometrics 1977:33:159-74.

38. Daley C. Chest radiography in the diagnosis of tuberculosis: specificity and accuracy. 39th World Conference on Lung Health of the International Union Against TB and Lung Disease. Paris, France: 2008:16-20.

39. Palaci M, Dietze R, Hadad DJ, et al. Cavitary disease and quantitative sputum bacillary load in cases of pulmonary tuberculosis. J Clin Microbiol 2007;45:4064-6.

40. Aderaye G, Bruchfeld J, Assefa G, et al. The relationship between disease pattern and disease burden by chest radiography, M. tuberculosis load, and HIV status in patients with pulmonary tuberculosis in Addis Ababa. Infection 2004;32:333-8. 